\title{
Resonant Channel Coupling in Electron Scattering by Pyrazine
}

\author{
Carl Winstead and Vincent McKoy \\ A. A. Noyes Laboratory of Chemical Physics, California Institute of Technology, Pasadena, California 91125, USA
}

(Received 18 December 2006; published 13 March 2007)

\begin{abstract}
Detailed investigation of the three low-energy resonances seen in electron scattering by the diazabenzene molecule pyrazine reveals that the first two are nearly pure single-channel shape resonances, but the third is, as long suspected, heavily mixed with core-excited resonances built on low-lying triplet states. Such resonant channel coupling is likely to be widespread in $\pi$-ring molecules, including the nucleobases of DNA and RNA, where it may form a pathway for radiation damage.
\end{abstract}

DOI: 10.1103/PhysRevLett.98.113201

PACS numbers: $34.80 . \mathrm{Bm}, 34.80 . \mathrm{Gs}$

Interactions between slow electrons and conjugated-ring molecules are strongly influenced by resonances. Disruption of DNA by subionization electrons [1-4], for example, appears to be mediated in part by resonances associated with the ring systems of the pyrimidine and/or purine nucleobases [3,5-7]. These resonances are commonly thought of as $\pi^{*}$ shape resonances arising from temporary trapping of the projectile in a vacant molecular orbital. However, as long ago as 1975, Nenner and Schulz hypothesized that the higher-lying shape resonances they observed in benzene and the azabenzenes "are probably mixed with core-excited shape resonances associated with low-lying excited states of the neutral" [8]. Subsequent observation [9] of the decay of the $4.8 \mathrm{eV} \pi^{*}$ resonance of benzene into electronically excited channels confirmed the reality of such mixing, and it is now routinely invoked in discussions of experimental results (e.g., [10,11]). However, such mixing has not been accounted for in calculations on elastic electron-molecule collisions, which therefore have implicitly assumed its effects on the elastic cross section are negligible. Indeed, many calculations have employed local-potential models of target polarization in which it may be difficult or impossible to account for resonant channel-coupling effects at all.

In recent calculations on the pyrimidine nucleobases uracil, thymine, and cytosine $[12,13]$, we observed a consistent pattern: Our resonance positions for the two lowestenergy and narrowest resonances (hereafter $R_{1}$ and $R_{2}$ ) were in reasonable agreement with measurements $[14,15]$, but our positions for the third and broadest resonance $\left(R_{3}\right)$ were too high. This was an unexpected result, because the most critical issue in obtaining good resonance energies is accounting for target polarization, which one might expect to be less important for $R_{3}$ than for $R_{1}$ and $R_{2}$ due to the decreased target-projectile interaction time. The same pattern was found in earlier calculations on benzene $[16,17]$ and the two-ring (purine) nucleobases adenine and guanine [18]; although we noted [17,18] the possible influence of coupling to core-excited resonances, we did not then explore it.

Here we investigate the observed trend in $\pi$-ring resonance energies by studying the diazabenzene molecule pyrazine (Fig. 1). Pyrazine is an ideal subject, because its high symmetry $\left(D_{2 h}\right)$ facilitates calculations and because $R_{1}, R_{2}$, and $R_{3}$ occur in distinct irreducible representations. As we will show, $R_{3}$ does indeed mix with configurations built on low-lying triplet excited states. That mixing contributes to a much larger polarization shift for $R_{3}$ than for $R_{1}$ and $R_{2}$, which are nearly pure single-channel (shape) resonances, and thus helps account for the past discrepancy between theory and experiment across $\pi$-ring molecules.

The relevant occupied and vacant $\pi$ molecular orbitals of pyrazine are shown in Fig. 2 and listed along with their conventional $D_{2 h}$ labeling in Table I. The orbital energies in Table I are canonical Hartree-Fock (HF) energies for the bound orbitals and static-exchange resonance energies for $\pi_{1}^{*}, \pi_{2}^{*}$, and $\pi_{3}^{*}$, in both cases computed in the 6-311+ $+\mathrm{G}(d, p)$ basis set as defined in GAMESS [19] and in the latter case obtained using the Schwinger multichannel (SMC) method [20]. (Full details of these calculations and others to be mentioned will be published separately.) Table I also lists the low-lying excited states of pyrazine computed at the single-excitation configuration-interaction (CI) level using GAMESS [19], again in 6-311 $++\mathrm{G}(d, p)$. A key feature, which is characteristic of benzene deriva-

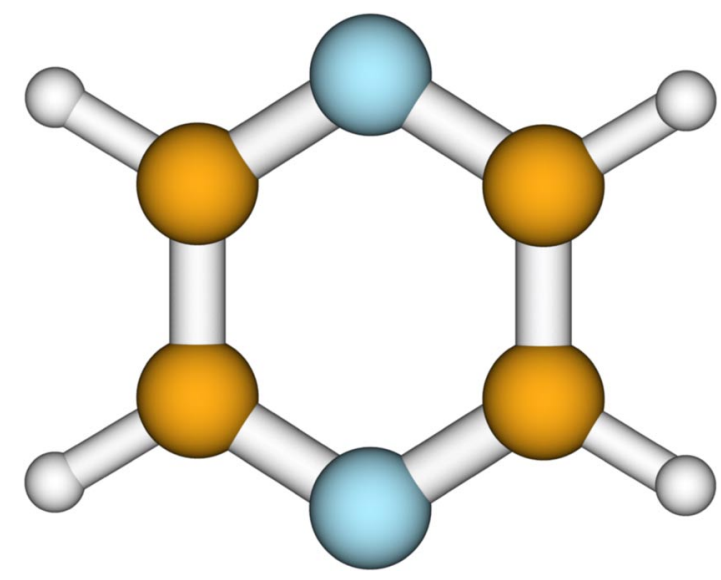

FIG. 1 (color online). Structure of the pyrazine molecule. Nitrogen is blue (light), carbon brown (dark), and hydrogen white. 


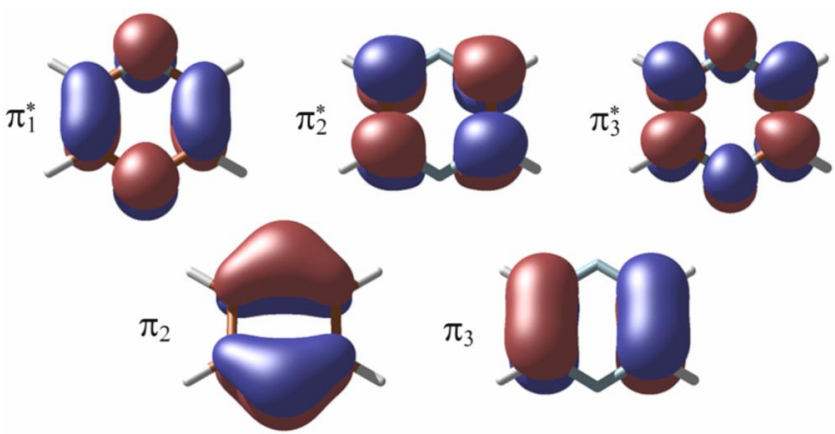

FIG. 2 (color online). Occupied and vacant $\pi$ molecular orbitals of pyrazine involved in the formation of low-energy electron-scattering resonances.

tives (and of unsaturated hydrocarbons generally), is the existence of low-lying triplet excited states. More particularly, we can note that the lowest-energy excited state, which arises from the $\pi_{2} \rightarrow \pi_{1}^{*}$ and $\pi_{3} \rightarrow \pi_{2}^{*}$ excitations, has ${ }^{3} B_{1 u}$ symmetry; therefore, if this state temporarily attaches an electron in the lowest empty orbital $\pi_{1}^{*}$, the resulting core-excited resonance has $B_{2 g}$ symmetry, the same symmetry as a shape resonance built on the ground electronic state by trapping an electron in $\pi_{3}^{*}$. Likewise, the third excited state $\left(\pi_{3} \rightarrow \pi_{2}^{*}\right)^{3} B_{2 u}$ can attach an electron in $\pi_{2}^{*}$ to form a $B_{2 g}$ core-excited resonance. Mixing between the $\pi_{3}^{*}$ shape resonance in the elastic channel and nearby core-excited states is therefore possible, although it remains to be seen whether it is a significant effect. Note that, in contrast, the lowest-energy core-excited resonance of appropriate symmetry to mix with the $\pi_{1}^{*}$ shape resonance is $1{ }^{3} B_{1 u} \otimes \pi_{3}^{*}$, while the lowest core-excited resonance that can mix with the $\pi_{2}^{*}$ shape resonance is $1^{3} B_{2 u} \otimes \pi_{3}^{*}$. Because of the larger energy gaps in these two cases, much weaker mixing can be anticipated.

Qualitative evidence for differential channel coupling in $R_{1}, R_{2}$, and $R_{3}$ can be obtained from small CI calculations on the pyrazine anion. We formed an active space from the three highest occupied HF orbitals and the five lowest +6 modified virtual orbitals (MVO's) [21]; this space includes $\pi_{2}, \pi_{3}$, and the three $\pi^{*}$ orbitals. Within this space, we carried out three CI calculations, allowing all excitations

TABLE I. Energies and symmetry labels for $\pi$ orbitals and low-lying excited states of pyrazine.

\begin{tabular}{lccc}
\hline \hline & \multicolumn{2}{c}{ Excited states } \\
Label & Energy $(\mathrm{eV})$ & Label & Energy (eV) \\
\hline$b_{3 g}\left(\pi_{1}\right)$ & -15.0 & $1{ }^{3} B_{1 u}$ & 3.41 \\
$b_{2 g}\left(\pi_{2}\right)$ & -11.9 & $1{ }^{3} B_{3 u}$ & 4.06 \\
$b_{1 g}\left(\pi_{3}\right)$ & -9.9 & $1{ }^{3} B_{2 u}$ & 4.08 \\
$b_{3 u}\left(\pi_{1}^{*}\right)$ & 1.8 & $1{ }^{1} B_{3 u}$ & 5.11 \\
$a_{u}\left(\pi_{2}^{*}\right)$ & 2.7 & $2{ }^{3} B_{1 u}$ & 5.13 \\
$b_{2 g}\left(\pi_{3}^{*}\right)$ & 8.7 & $1{ }^{1} B_{2 u}$ & 5.90 \\
\hline \hline
\end{tabular}

up to quadruples from reference configurations consisting of the HF neutral ground state times, respectively, $\pi_{1}^{*}, \pi_{2}^{*}$, and $\pi_{3}^{*}$. The results for $\pi_{1}^{*}$ and $\pi_{2}^{*}$ are nearly identical: The reference configuration has $\mathrm{CI}$ coefficient 0.97 , and the natural orbital of appropriate symmetry $\left(b_{3 u}\right.$ for $\pi_{1}^{*}, a_{u}$ for $\pi_{2}^{*}$ ) has an occupation number of 0.99 . In other words, they are both nearly pure single-configuration states. In strong contrast, the third CI calculation gives a coefficient of only 0.79 for the reference configuration, and the relevant orbital occupation numbers are $0.66\left(b_{2 g}\right), 0.44\left(b_{3 u}\right)$, and $0.30\left(a_{u}\right)$, indicative of strong mixing with (coreexcited) configurations in which $\pi_{1}^{*}$ and/or $\pi_{2}^{*}$ are populated. Although these are small calculations and lack proper scattering boundary conditions, they do strongly support the expectation that $R_{3}$ will behave much differently than $R_{1}$ and $R_{2}$.

To explore the effect of resonant channel mixing in detail, we used the SMC method to carry out staticexchange plus polarization (SEP) calculations of electron scattering by pyrazine in ${ }^{2} B_{3 u},{ }^{2} A_{u}$, and ${ }^{2} B_{2 g}$ symmetries. Again, we used 6-311+ $+\mathrm{G}(d, p)$ as the one-electron basis set. Three types of SEP calculation on $R_{3}$ will be contrasted. In the first, we treat polarization by allowing singlet-coupled excitations from the 15 valence occupied orbitals into the 43 lowest-energy MVO's, and we build from this "15-hole, 43-particle" (15h43p) set of 42electron states all 43-electron doublet states of appropriate symmetry. In the second, we employ only 30 MVO's as particle orbitals, but we include not only all singlet excitations from valence orbitals but also triplet-coupled excitations from the 6 outermost occupied orbitals $(15 h 30 p / 6 h 30 p)$. In the third, we selectively delete the configurations built on the triplet-coupled $\pi_{2} \rightarrow \pi_{1}^{*}, \pi_{3} \rightarrow$ $\pi_{1}^{*}$, and $\pi_{3} \rightarrow \pi_{2}^{*}$ excitations from the $15 h 30 p / 6 h 30 p$ calculation. The first calculation thoroughly incorporates the conventional picture of polarization as target relaxation, the second allows less for target relaxation but introduces virtual excitations of the target into the triplet manifold, and the third directly tests the importance of resonant channel coupling by removing the terms necessary to produce the lowest-lying core-excited shape resonances.

The results for ${ }^{2} B_{2 g}$ are shown in Fig. 3. Each calculation produces a clear $R_{3}$ resonance peak; the jagged structure at energies above $R_{3}$ is an expected computational artifact (pseudoresonances) due to our treating all triplet channels as closed, even though some are open at those energies. Even without any coupling to triplet virtual excitations, the resonance has shifted down significantly from the staticexchange energy for $\pi_{3}^{*}$; however, at nearly $6 \mathrm{eV}$, it is still far above the experimentally determined position [8], which is indicated by the horizontal bar in the figure. When we include triplet excitations in the closed-channel space, we obtain almost the correct location, with the resonance maximum now at $4.4 \mathrm{eV}$. If we then delete 
configurations built on the $\pi_{2} \rightarrow \pi_{1}^{*}, \pi_{3} \rightarrow \pi_{1}^{*}$, and $\pi_{3} \rightarrow$ $\pi_{2}^{*}$ triplet excitations - a matter of removing only 28 configuration state functions from the $15 h 30 p / 6 h 30 p$ calculation's 9037-function variational space-the resonance position shifts upward by $0.7 \mathrm{eV}$, from 4.4 to about $5.1 \mathrm{eV}$, and well out of agreement with experiment. Indeed, though not shown in the figure, removing just one resonancelike ${ }^{2} B_{2 g}$ configuration state function, namely, $\left(\pi_{3} \rightarrow \pi_{1}^{*}\right)^{3} B_{2 u} \otimes \pi_{2}^{*}$, shifts $R_{3}$ upward by fully $0.3 \mathrm{eV}$. Together, these results show not only that triplet excitations play a vital role in polarizing $R_{3}$ but more specifically that core-excited resonances built on the lowest triplet states mix strongly into $R_{3}$.

The $15 h 43 p$ and $15 h 30 p / 6 h 30 p$ results for $R_{1}\left({ }^{2} B_{3 u}\right)$ and $R_{2}\left({ }^{2} A_{u}\right)$ are shown in Fig. 4. For each of these resonances, nearly the correct position is obtained even without the triplet excitations, and including triplet excitations produces a smaller shift in resonance position than in the case of $R_{3}$. These results are fully consistent with $R_{1}$ and $R_{2}$ being nearly pure $\pi_{1}^{*}$ and $\pi_{2}^{*}$ shape resonances, for which target relaxation is the main polarization effect. Note, however, that the $15 h 30 \mathrm{p} / 6 h 30 \mathrm{p}$ calculations actually place $R_{1}$ and $R_{2}$ too low in energy - in fact, $R_{1}$ has become a bound state and disappeared from the scattering cross section. Such "overcorrelation" can arise because the configuration space, built on single excitations, introduces no dynamical correlation into the description of the neutral but does introduce some important $\pi \rightarrow \pi^{*}$ double excitations of the anion; for example, $\left(\pi_{3}\right)^{1}\left(\pi_{2}^{*}\right)^{1}\left(\pi_{3}^{*}\right)^{1}$ is a $B_{3 u}$ term doubly excited relative to the $\left(\pi_{3}\right)^{2}\left(\pi_{1}^{*}\right)^{1}$ configuration of $R_{1}$. Errors of a few tenths of an $\mathrm{eV}$ are thus reasonably expected at the current level of approximation.

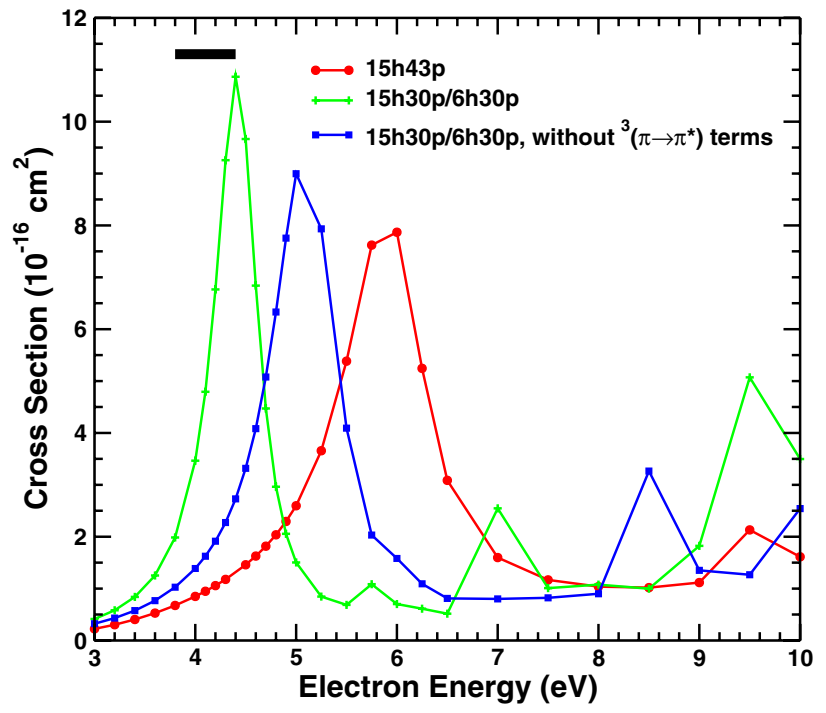

FIG. 3 (color online). Partial cross sections for electronpyrazine scattering in the resonant ${ }^{2} B_{2 g}$ symmetry obtained with different treatments of polarization (see text for discussion). The horizontal bar shows the measured resonance position (Ref. [8]).
Resonant channel coupling of the type affecting $R_{3}$ in pyrazine should be, as Nenner and Schulz posited [8], a general phenomenon affecting the high-lying resonances in benzene and the azabenzenes, as well as in such related molecules as the pyrimidine nucleobases of DNA and RNA. The failure of previous calculations $[12,13,17]$ to allow for excitation to low-lying triplet states thus accounts for much of the disagreement seen between the results of those calculations and related measurements [8,14,15]. Obviously, future calculations using all-electron methods should take the triplet states into account, either by including appropriate closed-channel terms in an SEP wave function, as was done here, or, perhaps better still, by explicitly including the lowest triplet states as open channels above their respective thresholds. On the other hand, it is difficult to see how such an effect, which leads to differential energy shifts among the $\pi^{*}$ resonances, can be incorporated in scattering methods that approximate polarization as an attractive local potential, unless perhaps by introducing an ad hoc energy dependence into the polarization potential.

It is interesting to speculate on the role that strong mixing between higher $\pi^{*}$ shape resonances and coreexcited resonances may play in promoting electron-impact dissociation of $\pi$-ring molecules, including the nucleobases. Computational evidence [22,23] supports the view that evolution has selected the nucleobases in part because they are stable against ultraviolet photolysis, due to conical intersections that rapidly return them from their lowest singlet excited states to high vibrational levels of the ground state whose excess energy is then dissipated thermally. Selective pressure against vulnerability to electroninduced damage would likely have been much weaker, and

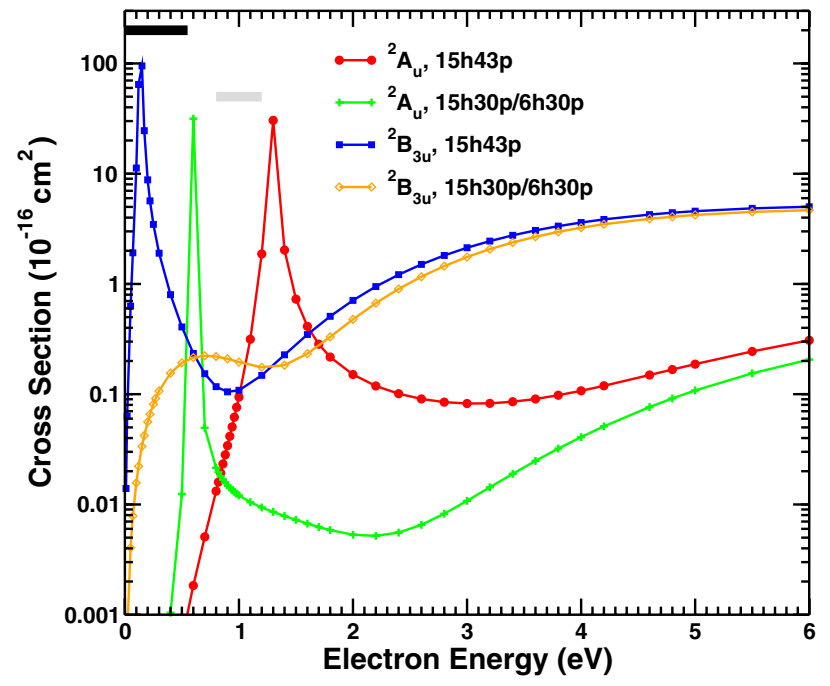

FIG. 4 (color online). Partial cross sections for electronpyrazine scattering in the ${ }^{2} B_{3 u}$ and ${ }^{2} A_{u}$ symmetries (see text for discussion). The horizontal bars (gray for ${ }^{2} A_{u}$ and black for ${ }^{2} B_{3 u}$ ) show the measured resonance positions (Ref. [8]). 
mixed resonances that decay readily not only into the elastic channel but also into triplet channels may provide a mechanism for damage to DNA. Triplet states have, in fact, been implicated in the formation of cyclobutylpyrimidine dimer lesions in DNA [24]. It seems possible, therefore, that the observed increase in DNA single-strand breaks at electron-impact energies above $4 \mathrm{eV}[2,4]$ is associated with the $\pi_{3}^{*}$ resonances of thymine and cytosine at, respectively, 4.05 and $4.50 \mathrm{eV}$ [14].

We gratefully acknowledge support of this work by the U.S. Department of Energy, Office of Basic Energy Sciences, and use of the computational resources of the Caltech-JPL Supercomputing Project. Figures 1 and 2 were prepared using MOLDEN [25].

[1] B. Boudaïffa, P. Cloutier, D. Hunting, M. A. Huels, and L. Sanche, Science 287, 1658 (2000).

[2] M. A. Huels, B. Boudaïffa, P. Cloutier, D. Hunting, and L. Sanche, J. Am. Chem. Soc. 125, 4467 (2003).

[3] F. Martin, P. D. Burrow, Z. Cai, P. Cloutier, D. Hunting, and L. Sanche, Phys. Rev. Lett. 93, 068101 (2004).

[4] R. Panajotovic, F. Martin, P. Cloutier, and L. Sanche, Radiat. Res. 165, 452 (2006).

[5] R. Barrios, P. Skurski, and J. Simons, J. Phys. Chem. B 106, 7991 (2002).

[6] J. Berdys, I. Anusiewicz, P. Skurski, and J. Simons, J. Phys. Chem. A 108, 2999 (2004).

[7] J. Berdys, P. Skurski, and J. Simons, J. Phys. Chem. B 108, 5800 (2004).

[8] I. Nenner and G. J. Schulz, J. Chem. Phys. 62, 1747 (1975).
[9] M. Allan, Helv. Chim. Acta 65, 2008 (1982).

[10] P. D. Burrow, J. A. Michejda, and K. D. Jordan, J. Chem. Phys. 86, 9 (1987).

[11] M. Allan, J. Electron Spectrosc. Relat. Phenom. 48, 219 (1989).

[12] C. Winstead and V. McKoy, J. Chem. Phys. 125, 174304 (2006).

[13] C. Winstead, V. McKoy, and S.d'A. Sanchez (unpublished).

[14] K. Aflatooni, G. A. Gallup, and P.D. Burrow, J. Phys. Chem. A 102, 6205 (1998).

[15] A. M. Scheer, K. Aflatooni, G. A. Gallup, and P.D. Burrow, Phys. Rev. Lett. 92, 068102 (2004).

[16] F. A. Gianturco and R. R. Lucchese, J. Chem. Phys. 108, 6144 (1998).

[17] M.H.F. Bettega, C. Winstead, and V. McKoy, J. Chem. Phys. 112, 8806 (2000).

[18] C. Winstead and V. McKoy, J. Chem. Phys. 125, 244302 (2006).

[19] M. W. Schmidt, K. K. Baldridge, J. A. Boatz, S. T. Elbert, M.S. Gordon, J.H. Jensen, S. Koseki, N. Matsunaga, K. A. Nguyen, S. J. Su, T. L. Windus, M. Dupuis, and J. A. Montgomery, J. Comput. Chem. 14, 1347 (1993).

[20] K. Takatsuka and V. McKoy, Phys. Rev. A 24, 2473 (1981); 30, 1734 (1984).

[21] C. W. Bauschlicher, J. Chem. Phys. 72, 880 (1980).

[22] A. Broo, J. Phys. Chem. A 102, 526 (1998).

[23] A. L. Sobolewski and W. Domcke, Phys. Chem. Chem. Phys. 6, 2763 (2004), and references therein.

[24] I. G. Gut, P. D. Wood, and R. W. Redmond, J. Am. Chem. Soc. 118, 2366 (1996).

[25] G. Schaftenaar and J. H. Noordik, J. Comput.-Aided Mol. Des. 14, 123 (2000). 